\title{
Key Emotional Values and Kawaii for Saudi Women
}

\author{
Barnawi Enayyah (MA15013@shibaura-it.ac.jp) ${ }^{1}$, Michiko Ohkura (Ohkura@sic.shibaura-it.ac.jp) ${ }^{2}$ \\ ${ }^{1}$ Graduate School of Engineering and Science, Shibaura Institute of Technology, Tokyo, 135-8548 \\ ${ }^{2}$ Department of Information Science and Engineering, Shibaura Institute of Technology, Tokyo, 135-8548
}

\begin{abstract}
In recent years, kansei engineering has become crucial in industrial fields. It works as a new value axis that differs from such conventional ones as functionality and price that have served as competitiveness sources in manufacturing. Based on the vast growth in the apparel industry and greater customer awareness and intelligent observation of various products, such a conventional value axis as functionality and price is insufficient to satisfy customer's needs. It is now crucial to study consumers' kansei or emotional values and build it within products. Kawaii value is one critical Kansei value in Japan. It is a Japanese word that is translated into English as cute, lovely, or adorable. However, it has a deeper meaning since it also represents an element of Japanese culture and not just a word with a single meaning. This research aims to define kawaii from Saudi women's perspective and identify the desired key emotional values for Saudi women and their importance compared to kawaii value. Using a qualitative research approach, semi-structured in-depth interviews were conducted and the grounded theory approach was used to analyze the data. Kawaii from Saudi women's perspective was defined and Saudi women's adaptation for kawaii value was clarified. The results showed that kawaii is not a critical value for Saudi women, however chic, elegance, and luxury are crucial desired emotional values for Saudi women.
\end{abstract}

Keywords: Saudi women, Key emotional values, Kawaii value, Social perception

\section{Introduction}

In recent years, kansei engineering has become crucial in industrial fields. The Japanese Ministry of Economy, Trade and Industry (METI) believes that a special type of positive economic value is created when industrial production is derived from Kansei (Industry and Initiative, 2015), which METI introduced as a new value axis that differs from such conventional ones as functionality and price that provide competitiveness in manufacturing. This new value axis is user/consumer kansei and probably includes the following feelings: enjoyment, sense of security, coolness, user friendliness, or empathy with manufacturer (Industry and fulfillment, 2015). Product design is also becoming more comprehensive because the customers are becoming more intelligent and market choices are becoming too many (Rajasekera and Karunasena, 2015). Therefore, affective production has become more complicated, and competing manufacturers are facing difficulty attracting a maximum number of customers. Thus, since product functions or prices are insufficient to attract customers, the kansei or emotional values of consumers must be incorporated within products.
Kawaii feeling is one important kansei value in Japan, as studies have proven that the existence of kawaii characters is one of the main reasons for the success of the digital content in Japanese games and animations (Belson and Bremner, 2004). Kawaii is a word with a positive meaning that can be translated into English as cute, lovely, or adorable (Belson and Bremner, 2004) and (Sakurai, 2009). However, it represents an important element of Japanese culture rather than just a word with a single meaning.

The term kawaii has been used in Europe and the rest of the world since the 1990s when such items of Japanese popular culture as manga, extravagant street fashions, and video games began to be exported (Koma, 2013). Nowadays, Japanese cartoons, animations, and drama have a significant increased presence among Saudi Arabian youth, which makes the term "kawaii" a familiar expression among Saudi youth especially those who are interested in Japanese animations. Thus, it is important to understand how kawaii is observed by people from countries that are distant from Japan, and have different cultural and social standards such Saudi Arabia.

In this study, we evaluated the desired key emotional values for Saudi women when purchasing fashion brands. In addition, we defined kawaii value from Saudi women's 
perspective. As a result, we identified the factors that influence the way Saudi women adapt different emotional values. Using a qualitative research approach, we performed in-depth interviews with Saudi women living in Japan, collected data, and analyzed it using The Grounded Theory Approach (GTA).

\section{Background}

In a previous study (Barnawi and Ohkura, 2016), we represented our attempt to clarify the similarities and differences in kawaii observation among genders and generations of Japanese and Saudi Arabians. However, the results raised an important research questions about the importance of kawaii value for Saudi women. Therefore, to investigate on this question, a group of Saudi females in Saudi Arabia were selected randomly and asked about their desired emotional values. Their answers varied between different values such chic, elegant, prestigious, etc. but none of them was "kawaii." In addition, results of two studies were found valuable for the process of this research. In 2015, a study was conducted to determine what motivates Saudi women to shop at western-style shopping centers; the results reflected that Saudi females believed that purchasing western-style goods helped them maintain their social prestige ( Alsubaie et al., 2015). After uniqueness and quality, the result of another study showed that emotional value was the third factor that influenced Saudi women to purchase luxury brands (Aqeel, 2012). The participants used a set of expressions to give their desired emotional values, including luxury, prestige, status, fashionable, and elegance.

The results from our investigation indicated that kawaii is not a critical value for Saudi females like it is for Japanese females; however, other values such chic, elegant, prestigious, etc. might be. This observation was a motivation to conduct this research.

\section{Method}

To satisfy the exploratory nature of this research and to get a deep understanding of research questions, a qualitative research method was used (Corbin and Strauss, 2015). To collect the data, we performed semi structured in-depth interviews which elicit answers close to users' real intentions by flexibly changing questions and topics based on their reactions as needed ( Hayakawa et al., 2013).

\subsection{Participants and interview questions}

The participants were four undergraduate Saudi women in their 20's living in Tokyo who are studying at different Japanese universities (scholarship students). All have been living in Japan for over two years and half, which means that they have sufficient knowledge about Japanese culture including kawaii. We performed two semi structured in-depth interviews, where two subjects were interviewed together as a focused group. The interviews were recorded using a voice recorder. The time interview took 1 hour and 25 minutes, and by the second interview took 53 minutes. The interview questions had two different themes, desired key emotional values and kawaii value. The participants were asked about different details regarding these two themes.

\subsection{Analysis}

We analyzed the data using GTA which enables the researchers to examine different topics and behaviors from many different angels, which helps in developing comprehensive explanations. It also can be used to get more information about old problems as well as to study new and emerging areas that are needed to be investigated (Corbin and Strauss, 2015). As an assisting tool to manage and organize the data, we used MAXQDA software. To analyze the data, we performed the following procedures:

1. transcribe the recorded voices into written text

2. translate the transcribed texts from Arabic to English

3. perform open coding which is an open process of giving proper concept to every piece of data

4. perform coding for concept development and elaboration where we refined the concepts that were defined in the open coding stage and elaborated more on them.

During the whole process, we mainly used memo writing and diagraming to develop the concepts in term of their properties and dimensions. We also performed coding for context to maintain the sensitivity of the action-interactions in different contexts. 


\section{Results}

\section{- $\quad$ The key emotional values for Saudi women}

We sorted the sub-concepts around the core concept and we defined the relationships between them. Then we made the diagram to illustrate the structure of the way Saudi women adapt their desired emotional values (figure 1).

Desired emotional values for Saudi women

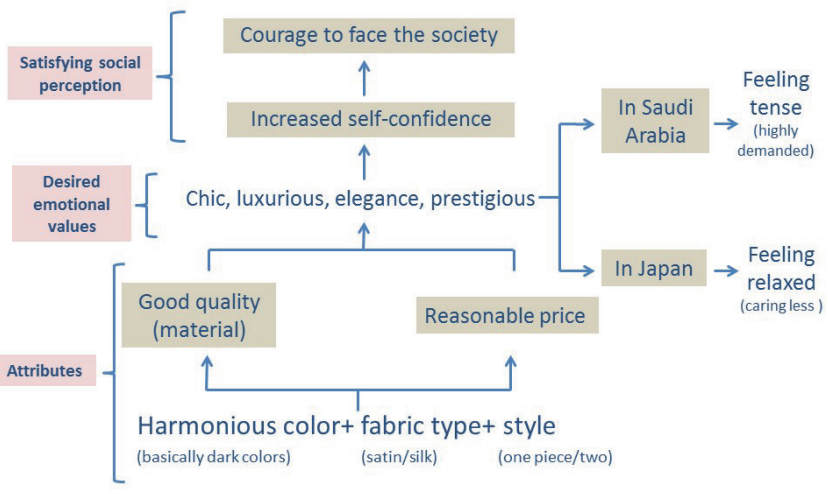

Figure 1. The structure of how Saudi women adapt their desired emotional values

The "desired emotional values" for Saudi women are chic, elegant, and luxurious. There are certain attributes that would make clothing chic, elegant, and luxurious such harmonious color (basically dark colors), fancy fabric type (such satin or silk), and certain style (for example: one or multi pieced clothing). These attributes should have a "good quality" and come with a "reasonable price." These values are very important for Saudi women and highly demanded "in Saudi" society, which put the women in "tense" toward these values because of the fear of the "social perception." They care about these values in special occasions (women-only community) the most. The idea of women-only community exists because of the nature of Saudi society where 1) women and men have separate environments; and 2) women wear abaya (traditional female garb) in public places. Therefore, the only chance for women to dress up freely (without the need of covering their hair or wearing abaya) is in women-only communities. Thus, they exploit this chance and try their best to be chic and elegant which considered as a high class values in Saudi. However, by being abroad such in this case "in Japan," the women tend to feel "relaxed" and care less because of the absence of social pressure. In general, they believe that embracing these values would "satisfy the social perception;" it would increase their "self-confidence," which in return will provide them with more "courage to face the society."

\section{- Kawaii value from Saudi women's perspective}

Figure 2 shows the general form of the way Saudi women adapt kawaii value.

Kawaii from Saudi women's perspective (in general)

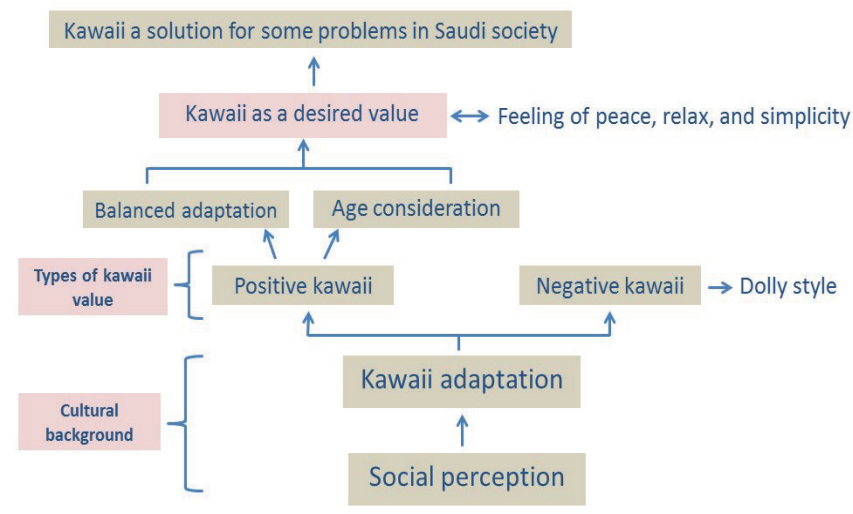

Figure 2. The structure of how Saudi women adapt kawaii value in general

Their adaptation for kawaii value is derived by the Saudi Arabian's social standards or in other words, by what the society might say about them "social perception." These standards are set by the Saudi Arabian "cultural background." For example, women in this study recognized kawaii as a value carrying childish meaning, while in Saudi culture it is unacceptable when adults try to dress or act in a childish way, thus they manage not to buy very noticeable kawaii goods in order not to get criticized by the society. According to Saudi women, there are two "types of kawaii value:" "positive kawaii" and "negative kawaii." "Positive kawaii" can be achieved by "age consideration" (meaning kawaii is for young girls, thus adults should adapt it with limitations) and "balanced adaptation" (meaning without over doing or over dressing). Dolly style is considered as "negative kawaii" because the lack of "balanced adaptation". The "positive kawaii" is the desired kawaii value for Saudi women that recalls the feelings of peace, relax, and simplicity. Consequently, "the desired kawaii value" can then be utilized as a solution for some problems in Saudi society such the problem of young girls acting ahead of their ages.

The results also confirmed that kawaii is not a critical value for Saudi women, however chic, elegance, and 
luxury are crucial desired emotional values for Saudi women.

\section{Discussions and Conclusions}

In this study, we evaluated the way Saudi women adapt kawaii and their desired emotional values through in-depth interviews and data analysis. Saudi women tied kawaii value with feeling "relaxed" and their desired emotional value with feeling "tense." Feeling relaxed is a key point for Saudi women, trying to be chic, elegant, and luxurious put them in tense all the time because of the fear of social perception. In contrast, kawaii value can cause and bring peace. It gave Saudi women the feeling of peace and made them believe that importing kawaii value to Saudi would contribute in solving some problems in Saudi society. If kawaii is adapted as a culture, the feeling of peace that is evoked by kawaii should reflect on one's mentality and personality, which would reduce the violence and negativity. The results showed that social perception have significant impact on the way Saudi women adapt kawaii and their desired emotional value. They really care about what other women in their society might say about them. They are willing to be chic and elegant at any expense just for the sake of increasing their self-confidence, which in return will give them more courage to face the judging society. They strictly maintain the way they adapt kawaii value. Their adaptation for kawaii is limited to what is acceptable in their culture in order not to get criticized by the society. The pyramid in figure 3 illustrates the hierarchy of how Saudi women adapt different emotional values. The cultural background works as a base line that sets and controls society's standards, which forms the way individuals perceive each other. Thus, these individuals tend to adapt different emotional values in a way that fits the social standards and satisfy the social perception.
However, the authors believe that the impact of social perception might not last for the coming generations. Nowadays, Saudi society is exposed to globalization, and the traditional way of thinking is gradually decreasing.

\section{References}

Alsubaie, H., Valenzuela, R. and Adapa, S., "The Advent of Western-Style Shopping Centers and Changes in Saudi Women's Purchasing Behavior" In Emerging Research on Islamic Marketing and Tourism in the Global Economy, 2015.

Hayakawa, S., Ueda, Y, Go, K., and Takahashi, K., "User Research for Experience Vision", Human-Computer Interaction. Berlin: Springer. 2013.

Koma, K., "Kawaii as Represented in Scientific Research: The Possibilities of Kawaii Cultural Studies". (pp. 103-117). 2013.

Aqeel, A.,'Factors Influencing Saudi Women to Purchase Luxury Fashion Brand", International Conference on Management, Behavioral Sciences and Economics Issues. Malaysia. 2012.

Barnawi, E., and Ohkura, M., "Evaluation of levels of kawaii ribbons among genders and generations of Japanese and Saudi Arabians", APD2016. Florida. 2016.

Belson, K., and Bremner, B. Hello Kitty: The Remarkable Story of Sanrio and the Billion Dollar Feline Phenomenon. New Jersey: John Wiley \& Sons. 2004.

Corbin, J., and Strauss, A., Basics of Qualitative Research; Techniques and Procedures for Developing Grounded Theory. London: SAGE. 2015.

Kansei Initiative from materialistic fulfillment to emotional fulfillment, Ministry of Economy Trade and Industry, http://www.meti.go.jp/english/policy/ mono_info_service/mono/kansei2009/(retrieved December 2015)

Kansei Value Creation Initiative, Ministry of Economy Trade and Industry, http://www.meti.go.jp/english/information/downloadfiles/PressRelease/08 0620KANSEI.pdf (retrieved December 2015)

Rajasekera, J., and Karunasena, H., "Apparel Design Optimization for Global Market: Kansei Engineering Preference Model”, Intrnational Conference on Biometric and Kansei Engineering (pp. 119-126), Japan. 2015.

Sakurai, T., Sekai Kawaii Kakumei, Tokyo: Ascii Media Works. 2009. (in Japanese)

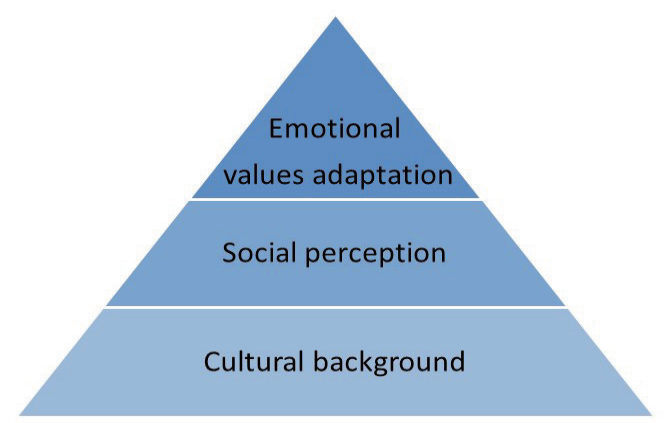

Figure 3. Emotional values adaptation hierarchy 